\title{
Optimization of Operations by Simulation-A Case Study at the Red Cross Flanders
}

\author{
Karen Moons, Helena Berglund, Valerie De Langhe, Katrien Kimpe, Liliane Pintelon, \\ Geert Waeyenbergh
}

Research Group Sustainable Engineering, KU Leuven, Leuven, Belgium

Email: Karen.Moons@kuleuven.be

How to cite this paper: Moons, K., Berglund, H., De Langhe, V., Kimpe, K., Pintelon, L. and Waeyenbergh, G. (2016) Optimization of Operations by Simulation-A Case Study at the Red Cross Flanders. American Journal of Industrial and Business Management, 6, 1001-1017.

http://dx.doi.org/10.4236/ajibm.2016.610096

Received: September 12, 2016

Accepted: October 16, 2016

Published: October 19, 2016

Copyright $\odot 2016$ by authors and Scientific Research Publishing Inc. This work is licensed under the Creative Commons Attribution International License (CC BY 4.0).

http://creativecommons.org/licenses/by/4.0/ (c) (i) Open Access

\section{Abstract}

The Blood Service at the Belgian Red Cross-Flanders is responsible for blood collection in Flanders (Belgium). One of their missions is guaranteeing a constant and sufficient supply of safe blood products. This is a critical public health need, since the blood products can save lives of victims from traffic accidents or in the event of major blood losses in hospitalized patients. The main objective of this project is optimizing the operations flow in donor centers, in such a way that the waiting time for donors is minimized and that the donor center occupation or productivity is maximized. In this case study, the flow of three types of donations is investigated. Blood and plasma are donated in all donor centers (i.e. 11 donor centers in Flanders), while blood platelets are collected in only six donor centers. Based on data collected from the 11 donor centers in Flanders, a simplified simulation model was developed, which can be used to optimize the operations flow based on the expected number of donors and their moment of arrival at the donor center. The simulation model is built in Enterprise Dynamics 9.0 simulation software. The input data in the model are data that have been collected in collaboration with the Belgian Red CrossFlanders. Different scenarios will be analyzed to gain insight in the impact of small changes in the input parameters on the performance of the flow. In this paper, a gap analysis is conducted to identify extra data needs. With these additional data, a more detailed model can be constructed to test the scenarios, and a dynamic planning tool will be developed to rely on when setting up the capacity of the donor center in order to find a scenario with the most optimized flow.

\section{Keywords}

Optimization, Simulation, Operations Flow, Blood Supply Chain 


\section{Introduction}

\subsection{Problem Formulation and Objectives}

The Belgian Red Cross-Flanders, part of the international Red Cross and Red Crescent Movement, is an independent volunteer organization with a threefold mission which consists of stimulating self-reliance, providing assistance in the event of emergencies and excelling in blood supply. The department Blood Service is responsible for ensuring a continuous and sufficient supply of safe blood products that will be distributed to the majority of hospitals in Flanders [1]. Their activities are based on two principles: voluntary unpaid donations and national self-sufficiency in blood products [2]. In Flanders, the Blood Service is operating in 11 fixed donor centers and about 770 mobile sites to make donating more convenient for donors.

The availability of sufficient quantities of safe, high-quality blood is a critical public health need as it is helping doctors to save lives. Matching supply and demand for blood, however, is not straightforward because of several external factors. Seasonality, regional trends and other unforeseen circumstances, like flu epidemics or crises, may impact the availability and need for blood. This matching process is further complicated by the limited shelf life of blood products. As a consequence, a constant supply of blood is needed as well as a good inventory policy in order to reduce blood shortages, which may cause increased mortality rates and hence high costs for society [3]. As Kendall has noted, "Blood is essential for surgical procedures and medical therapy. It is critical that blood be available to everyone who needs it, and yet it is also important that little of this valuable and limited resource is wasted" [4]. Several components (i.e. blood, plasma, blood platelets, etc.) can be extracted from the whole blood, and each component performs a specific function in the human body. Different blood products will be used in different situations when treating patients. Red cells are used for surgical patients or other patients in the event of major blood loss, for anemic patients and for premature infants; platelets are used for cancer patients as well as for surgical patients and patients in the event of major blood loss; and plasma is used for surgical patients, patients in the event of major blood loss and for treatment of liver diseases and burn injuries [2].

In Belgium, $70 \%$ of the Flemish people will need a blood transfusion during his/her lifetime, while only $3 \%$ are donating. Each year 350,000 bags of blood are donated in Flanders. In the production laboratory, these blood bags are transformed into 600,000 deliverable high-quality blood products which are transferred to the majority of hospitals in Flanders [5].

The Blood Service at the Red Cross-Flanders aims to improve the efficiency of their donor flow. External factors and variability complicate the organization of donors and resources at donor centers. As a consequence, it is difficult to ensure an optimal utilization of the resources, which may result in queues (i.e. waiting lines) for donors and under- or overutilization of the resources at certain moments of the day. The Blood Service wants to improve their operations with focus on the comfort and satisfaction of donors. 
To gain insights in the performance (e.g. queueing problems) of the donor centers, an operations analysis was conducted about the current situation. This in order to build a simulation model. Simulation enables to model the donor flow and test several scenarios based on changing the input parameters, without experimenting or spending any money on the work floor. In this paper, we present the efforts that were done to develop a first simulation model that visualized the donor flow in a reference donor center.

The outcome of the operations analysis indicated that various parameters are influencing the performance of the donor flow. Seasonality in the arrival pattern of donors, the location of the donor center, launching a campaign, the capacity of the resources, the ratio of new and experienced donors, etc. are used as input parameters in a simulation model. The simulation software Enterprise Dynamics 9.0 was used to build a first, simplified model that represents the current donor flow. As reference donor centers for the model, the data from donor center Ghent and Geel were used as input parameters. Based on this model, a gap analysis was conducted to identify additional data needs to build a more detailed simulation model. The final objective of our study is to develop a dynamic planning tool on which the Blood Service can rely on when setting up the capacity of the donor center in terms of both personnel and beds. In the ideal case it would be possible to have a dashboard that can show the optimal organization of resources related to the parameters of the expected situation.

Building the simulation model under the strategy of lean thinking can help to eliminate the waiting times, have a more efficient utilization of the resources, and an efficient use of space. Healthcare quality and costs depend on delivery processes, which often include unnecessary or inappropriate steps that do not contribute to the value of patient care [6]. Lean management, commonly associated with the Toyota Production System (TPS) in manufacturing, is a relatively new concept to healthcare. In 2005, the American Institute for Healthcare Improvement [7] advocated using lean management as it began to show promising results (i.e. maximizing value and eliminating waste) in healthcare. Lean thinking is based upon two concepts: the reduction of costs through the elimination of waste (i.e. activities that do not add value to the product) and the full utilization of workers' capabilities [8]. D'Andreamatteo, Ianni, Lega and Sargiacomo [9] review the diffusion of lean in healthcare. Various journals (especially medical and nursing journals) are interested in lean thinking in order to improve operational efficiency, clinical outcomes of care processes and well-being at work. In a healthcare service environment, the patients constitute the primary flow. It is therefore necessary to incorporate a sociotechnical approach to the healthcare system and the design of healthcare facilities. The design can have a large impact on efficiency and outcomes [10], because multiple stakeholders, many outcomes and flows (patients, staff, family and friends, medications, supplies, equipment and information) are interacting. The Virginia Mason Hospital in Seattle used the Production Preparation Process (3P) method, which is part of the lean design process, together with the seven flows of medicine for optimizing their facilities design to encourage collaboration between the stakeholders early in the design process [11] [12]. However, Nicholas [6] noted that "lean is no 
panacea, but it can significantly reduce waste". In order to implement the lean principles, an organizational culture should be created that is receptive to lean thinking. When applied rigorously and throughout the entire organization, lean management strategies can help improve processes, reduce costs and increase satisfaction among patients and employees.

The remainder of this paper is organized as follows. In the next section, the donor flow at the Red Cross-Flanders is described. Section 2 focuses on the use of simulation as a tool to optimize the donor flow. An operations analysis was done by the Blood Service, since they were observing queueing problems in the flow. Section 3 provides the process of building the first simplified simulation model and implementing the available data. It is crucial to check for model validation and verification since simulation models are simplifications of reality. The results are provided in Section 4 . This section also conducts a gap analysis to identify the missing data and provides recommendations in order to build the dashboard and test different scenarios in the second phase of the project. The conclusion and suggestions for future work are presented in Section 6 .

\subsection{Operations Flow}

The operations or donor flow is divided into five steps. The sequence of these steps is similar in the 11 donor centers of the Red Cross-Flanders, but they differ in the types of donations that can be made. In the donor centers, mainly three types of donations are collected. Blood and plasma can be donated in all donor centers, whereas blood platelets in only six donor centers. In this case study, a simplified simulation model is built by analyzing two reference donor centers. In Ghent, the three types of donations are collected, while the donor flow in Geel is not equipped to donate blood platelets.

Figure 1 shows a schematic representation of the five steps that are performed during a visit at the donor center, regardless of the type of donation. The main objective is to dynamically optimize this donor flow by improving the customer (or donor) service and by using the resources more efficiently during the day, week and year. The flow starts when a donor enters the donor center and ends with the donation collected in step 4 . This blood bag will be further processed into end products in the production laboratory, from where it will be distributed to the hospitals in order to help patients in need of these products. The Blood Service is continuously improving their customer service in every step of the donation process to offer qualitative blood products to patients and to deliver optimal quality to hospitals [13].

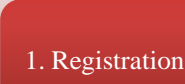

Figure 1. Donor flow at Red Cross Flanders. 
In this paragraph, the different steps of the donor flow, displayed in Figure 1, will be explained, which constituted the main building blocks in the simulation model. In between the steps in the donor flow, a queue can occur depending on the number of donors in the flow and the capacity of the resources. The donor flow starts when the donor arrives at the donor center. The donor details are recorded at the registration desk by scanning the electronic identity card. The person at the registration desk prints a medical questionnaire and the donor gets a post-donation card with a unique number for each donor and donation. In the second step, the donor chooses one of the available spots where he/she can quietly answer the medical questionnaire. The questionnaire is designed to elicit information on the state of health and lifestyle of the donor, as well as to identify any medical procedures or potential risks. In step 3, the doctor is examining the donor by recording the weight of the donor and by taking blood pressure and pulse. The hemoglobin level is measured for new donors and for donors whose hemoglobin level at the previous donation was below the acceptable limit. Based on this medical checkup, the answers to the questionnaire and any additional oral questions, the doctor decides if the donor is allowed to donate or not. The first three steps are the same in the donor flow in each donor center, independent of the type of donation. In the fourth step, the donor is assigned to a bed for the actual donation. From this step, the donor flow is split up in three separate flows: blood, plasma and platelets collection. At the beginning or during the first minutes of the actual donation, the donor is registered in the blood information system by scanning the post-donation card and the materials and products used for the donation. After the donation, a compression bandage is put on the arm to cover the small puncture wound. The last step in the donor flow gives the donor the opportunity to relax and have a drink in the assigned refreshment area (i.e. donor corner).

\section{Simulation}

The healthcare sector is becoming more competitive. Hospitals are aiming at delivering efficient and effective healthcare which requires high quality medical care. "In healthcare, efficiency means a better allocation of scarce resources which will result in a higher overall quality of healthcare" [2]. Simulation is a useful tool to make operations more efficient, and it is defined as "the process of designing a model of a real system and conducting experiments with this model for the purpose of understanding the behavior of the system and/or evaluating various strategies for the operation of the system" [14]. In this paper, Enterprise Dynamics 9.0 simulation software is used to apply discrete-event simulation to build the donor flow and to investigate the impact of operational changes at the Red Cross-Flanders [15]. It is an object-oriented simulation platform that allows to model, visualize and monitor dynamic flow process activities. The software enables you to create insights in the donor flow, evaluate potential resource investments, answer what-if questions by modeling several scenarios, and to estimate the impact of external factors and variable process times on the performances of the flow [16]. 
The main objective of this project is improving the operational flow in a donor center by using simulation in order to find the optimal flow in different situations. An efficient donor flow can be obtained by optimizing the utilization rate of the resources and decreasing the waiting times for donors which will result in the collection of safe, qualitative blood products for saving patient lives. However, the results of the operations analysis (see Section 1.1) showed that various parameters are influencing the performance of the donor flow. A simulation model enables the Blood Service to change the input parameters and answer what-if questions to find the optimal flow in each situation. Moreover, the visualization of the donor flow, which can also be displayed in 3D, is a strong advantage when using simulation to convince medical experts of the benefits.

At first, simplified simulation model, corresponding to the current donor flow, is built in cooperation with employees and medical experts at the Blood Service. Involvement of the personnel in the healthcare sector is vital so that the model under development can be validated based on their understanding of the operations flow [2]. Moreover, monitoring the current flow and identifying potential improvements by using realistic visualizations (3D viewer) is important to convince the medical experts of the advantages of the project for both donors and the resource utilization. The model allows the user to analyze how the output is affected by (changing) the input parameters and to identify the bottlenecks in the donor flow. Answering what-if questions by analyzing different scenarios (without spending any money on utilizing more personnel for example) in order to find the optimal flow in different situations, makes simulation an attractive tool to investigate the problem from different perspectives and to analyze trade-offs in healthcare systems [17].

Keep in mind that simulation models are simplifications and that sometimes it might be difficult to guarantee their validity. It is crucial that the model reflects the behavior of the real donor flow. Model validation and verification (see Section 3.4) will check for this. In order to build a valid simulation model, data should be implemented in the model. The Blood Service is collecting data in a blood information system (CTS Serveur software package by Haemonetics). By applying goodness-of-fit tests, probability distributions are selected that best fit the data. However, lack of data is a well-known problem in healthcare. Additional data should be collected to obtain accurate simulation results in order to develop the dynamic planning tool.

\section{Modelling}

\subsection{Operations Description}

The core of the model to build is to be found in the operations layout (summarized in Figure 1). The donor flow was observed in collaboration with experts of Blood Service at the Red Cross-Flanders in order to create knowledge on the donation process in a donor center. Each donor goes through the same five steps in the donor flow. Depending on the type of blood product they are donating, the duration of the actual donation (step 4) will take more or less time. 


\subsection{Data Collection}

The Blood Service is recording data about the donor flow in the blood information system (i.e. CTS Serveur software package). These data are converted to useful information reports using a business intelligence database (cognos database), in which the number of arriving donors per hour and per day can be retrieved, as well as the time the donors are spending in the donor flow (i.e. end-to-end donation time). However, only two time registrations are shown in this database: (1) the moment at which the medical questionnaire is printed at the registration desk, which is assumed to be the start of the donor flow (step 1), and (2) the start of the actual donation (step 4), which is recorded at the moment of scanning the post-donation card in the blood information system during the first minutes of the donation. This second registration moment is not pre-defined and hence not accurate. For the donation of blood, this registration should happen prior to the start of the actual donation, while plasma and platelets donations already start and the donation is registered during its first minutes. The remaining duration of the actual donation is estimated by medical experts to be 6,40 and 75 minutes for the donation of blood, plasma and platelets respectively. Furthermore, the blood information system provides important data on the busiest times (i.e. peak hours) and least congested times (i.e. trough hours) of the day. At these times, the arrival pattern of donors will be different. Changing this parameter in the simulation model will affect the performance of the donor flow. The objective of this project is to find the most optimal flow, such that the Blood Service is capable of adapting the input parameters in real-time to the arrivals of donors by optimizing the organization (utilization rate of the resources) of the donor center. As a result, the waiting times should decrease.

As mentioned in paragraph 1.1, Ghent and Geel are serving as the two reference donor centers in this study. Data from available manual data collections in the 11 donor centers can be used to be implemented in the simulation model. Some data, however, are center-specific, such as arrival times of the donors. In the first, simplified simulation model, the exact arrival times were implemented in order to mimic the behavior of the real donor flow. Later on, probability distributions will be selected that best fit the arrival data. Ghent is one of the largest cities in Flanders. The donor center in Ghent is equipped to collect the three types of donations. In 2015, 24,304 donations were collected, which is higher than in an average Flemish donor center. They collected $18.6 \%$ of the blood donations, $13.8 \%$ of the plasma donations and $22.5 \%$ of the platelets donations across all donor centers in Flanders. The donor center in Ghent was also selected because it serves as a reference center in a pilot study, approaching this project. The donor center in Geel is representative for all donor centers that are not collecting platelets donations. In 2015, a total of 7,804 donations are collected, which can be split up to $4.5 \%$ of the blood donations and $6.6 \%$ of the plasma donations. It is assumed that the donor flow works similarly in the 11 donor centers in Flanders, except for the type of donations they are collecting.

The available data at the Red Cross-Flanders, collected either by the blood information system or manually by the employees, are implemented in a first, simplified simu- 
lation model. These data represent times of the day and should be converted to times expressed in minutes. For example, 9:05 is equivalent to 5 minutes, because we only take into account the opening hours (i.e. 9 am till $7.30 \mathrm{pm}$ ) of the donor center. Another example, 15:45 can be expressed by 405 minutes $(15 * 60+45-9 * 60)$. To capture variability in the data, AutoFit is used to find the probability distributions that best fit the observed data. AutoFit is a goodness-of-fit test integrated in Enterprise Dynamics, which determines whether a certain data set can be represented by a certain distribution based on shape, mean and standard deviation. By default, the Anderson-Darling test is used [16]. Table 1 summarizes the collected data and distributions for each step in the donor flow.

\subsection{Building the Simulation Model}

The simulation model, as shown in Figure 2, represents a good visualization of the current flow at the donor centers at the Red Cross-Flanders. The structure is equivalent to the donor flow displayed in Figure 1. In the simulation software Enterprise Dynamics 9.0, atoms (i.e. building blocks) are representing each step of the donor flow.

Table 1. Probability distributions of the donor flow.

\begin{tabular}{ccc}
\hline Donor flow & Datapoints & Distribution (minutes) \\
\hline Registration desk & 53 & Erlang $(2.55,4.00)$ \\
Medical questionnaire + waiting & 26 & Weibull $(5.06,1.80)$ \\
Doctor & 574 & Weibull $(3.91,2.50)$ \\
(Queue before plasma) & 22 & Erlang $(3.79,2.00)$ \\
(Queue before platelets) & 14 & Erlang $(6.31,1.00)$ \\
Actual donation blood & 0 & Normal (6.00,1.00) (assumption) \\
Actual donation plasma & 42 & Logistic $(42.82,9.30)$ \\
Actual donation platelets & 28 & Normal (77.91, 66.59)
\end{tabular}

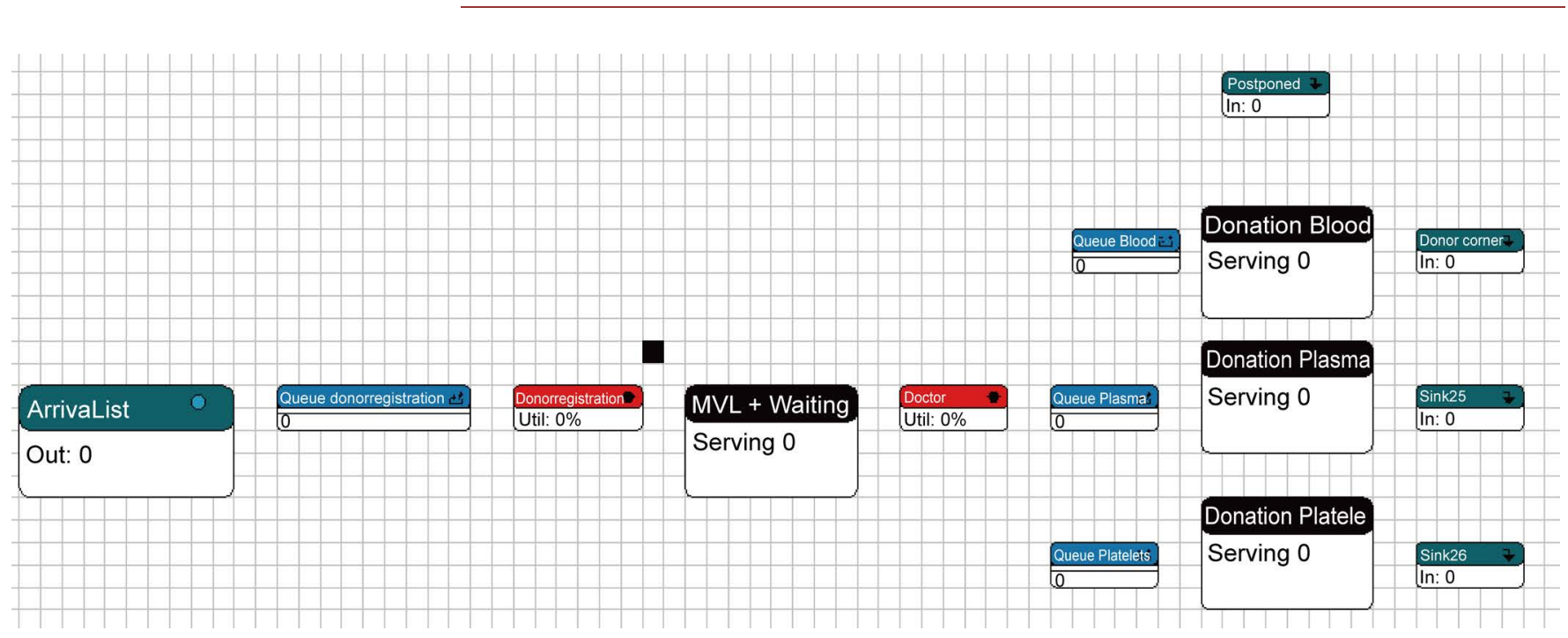

Figure 2. First simulation model in enterprise dynamics 9.0. 
The basic atoms used in this model are Source, Queue, (Multi-) Server and Sink atoms. The simulation model can easily be adapted to a flow without platelets donations, as these are only collected in six donor centers.

The donors arrive at the donor center according to a random arrival pattern. Data regarding arrivals at the donor center in Ghent were used and are converted to data lines in the ArrivalList atom. This atom creates donors based on the pre-defined list, which contains data on the arrival time, a name, the arrival quantity and a channel through which the incoming donors are sent by using three labels. As such, a distinction can be made between the three types of donations. When entering the donor center, the donor may have to wait in a queue before being served by the person at the registration desk (i.e. entering queue). At the registration desk, which is represented by a Server atom, one employee is occupied with registering all arriving donors. On average, this activity takes 2.55 minutes and AutoFit suggests an Erlang distribution (see Table 1). After the registration, the donor gets time for completing the medical questionnaire. At the reference donor center, five spots are arranged for filling in this questionnaire quietly. These spots are depicted as a Multi-Service atom, because the donors can do this activity simultaneously with four other donors. In the simplified model, the time for completing the questionnaire (Weibull distribution with an average of $5.06 \mathrm{mi}$ nutes) also includes the waiting time for entering the doctor's office. Hence, no Queue atom is built in front of step 3. Based on the medical examination, the answers to the questionnaire and any additional oral questions, the doctor will decide whether or not the donor can donate. This activity takes on average 3.91 minutes and is Weibull distributed. Donors who are not allowed to donate (i.e. postponed donors) leave the flow and do not continue to the actual donation step. Donors who are allowed to donate are assigned to a bed, if any bed is available. Otherwise the donor waits in a queue. From now on, the flow is split up in three separate flows for the three types of donations. Hence, it is important to send the donors by the right label as these are indicating the type of donation. For example, donations of blood are labeled in the ArrivalList atom to go through the first channel, while plasma and platelets donors go through the second and third channel respectively. Once the donor is installed on the bed, the actual donation can start. Multi-Service atoms represent the beds for donation. In this case, there are three beds for blood donations, seven beds for plasma donations and four beds for platelets donations. The duration of the actual donation is assumed to be 6,40 and 75 minutes for blood, plasma and platelets respectively. However, the collected data for plasma and platelets donations follow a logistic and normal distribution with an average of 42.82 and 77.91 minutes, respectively. For the actual donation of blood, there are no data available, so a normal distribution with an average of 6 minutes is assumed. When the donation ends, a compression bandage is put on the small puncture wound and the donor leaves the bed. In the donor corner (Sink atom), he/she can have a drink and relax before going home. This step does not have any impact on the efficiency of the donor flow, but it serves to satisfy the donors.

In the first model, Queue atoms are used to model waiting lines. However, the Red 
Cross-Flanders collected data on the time that donors are spending in a queue before being assigned to a bed in step 4 . Therefore a second simulation model has been developed in which the queues are replaced by Multi-service atoms, representing the observed waiting times. By comparing the two simulation models and the observed data, one can check whether the first simulation model is valid (see paragraph 3.4). The observed data suggest an Erlang distribution with an average waiting time of 3.79 minutes for plasma and 6.31 minutes for platelets. For blood donations, there are no data available on queueing. The first simulation model suggests that this queue is always empty. Hence no Multi-service atom has been included in the flow of donors in the second simulation model (see Figure 3).

\subsection{Validation and Verification of the Simulation Model}

When developing a simulation model, it is crucial to do a validation and verification. The Red Cross-Flanders will make important decisions based on the results of the model, which will affect the employees and the donors. Model verification is often defined as "ensuring that the model and the implementation are correct", while model validation determines whether "the model within its domain of applicability possesses a satisfactory range of accuracy consistent with the intended application of the model" [18]. Unfortunately, there is no set of specific tests that can easily be applied to determine the correctness of the model [19].

The simulation model is verified by investigating whether the model is built in the right way. The models in Figure 2 and Figure 3 are built to specifications by implementing the observed data in the different steps of the donor flow. After running the model for one day (i.e. opening hours between 9 am and $7.30 \mathrm{pm}$ ), no errors occurred and all the donors who arrived at the donor center also left the donor corner. As such, we can secure the right operation of the simulation model in regard to the functional processes.

Model validation is important to ensure that the right model is built to meet the intended purpose of the project. Three tests were executed to check for model validation.

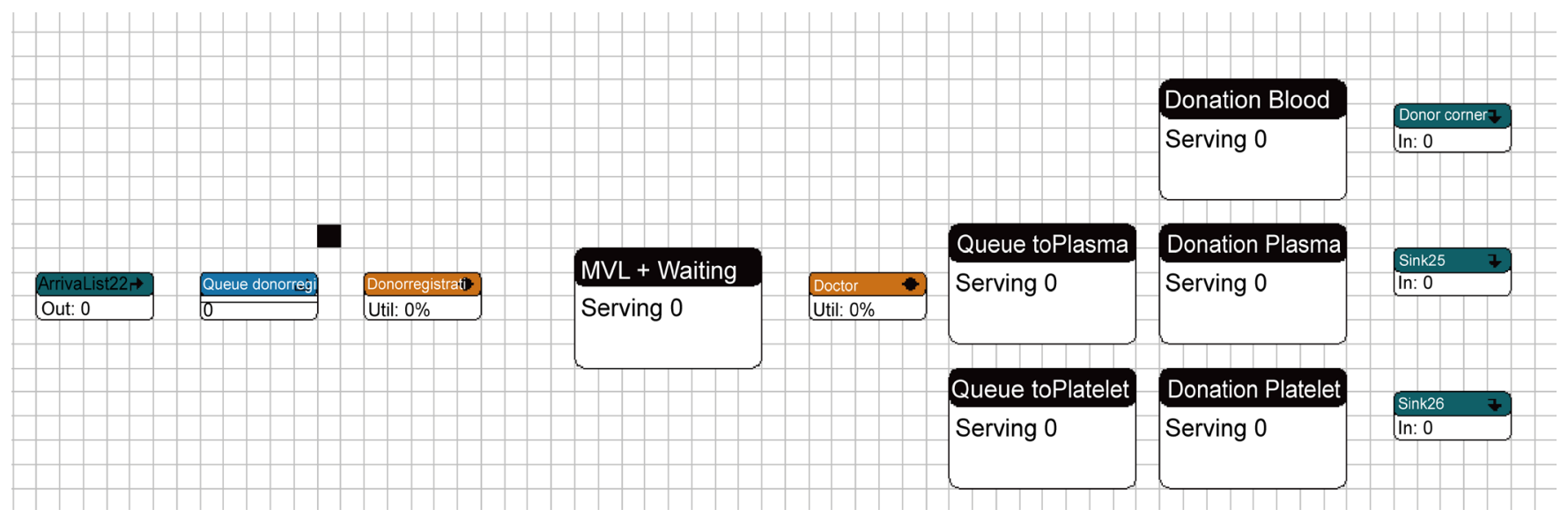

Figure 3. Second simulation model in enterprise dynamics 9.0. 
In the first test, the ArrivalList atom was observed to make sure that the donors arrive at the donor center according to the observed data. The second validation method monitors the Sink atoms according to the principle "what comes in, goes out". The 112 incoming donors also leave the model, labeled by the three types of donation. By using the ArrivalList atom, label values were assigned to the incoming donors referring to the type of donation. A third test is performed to check whether the model corresponds to reality by comparing the average end-to-end donation time (i.e. the time a donor spends in the donor flow). Table 2 shows the average end-to-end donation time in the two simulation models and in the observed data. The calculated end-to-end donation time based on the observed data is not yet very accurate because the blood information system only collects data at two time registrations (i.e. printing of medical questionnaire and start of actual donation). The estimated durations for the actual donations are added to these times. In Table 2, column 2 should approximate column 4, because the second simulation model represents the waiting times as observed in the data. Due to lack of data, the end-to-end donation times differ slightly. The two simulation models in column 3 and 4 also indicate different end-to-end donation times. The time difference between the first and second model is 5 minutes for plasma and 7 minutes for platelets, which is due to the pre-defined waiting time in front of the actual donation in the second model. For blood donations, however, there is no difference between the models because there is no queue. The first simulation model indicates that the current end-to-end donation time could be reduced.

\section{Results and Recommendations}

\subsection{Results}

This section presents the results obtained by running the first simulation model. Based on Table 2, the first model is selected to further investigate because the end-to-end donation time is lower which may point at a potential optimization of the current donor flow. However, it should be noted that the human resource team is not yet accurately modeled. In Enterprise Dynamics 9.0 simulation software, four techniques are available to present the results. The results have been obtained by doing ten times a simulation run of 12 hours (i.e. donors can arrive to the center between 9 am and $7.30 \mathrm{pm}$ ).

A first method for interpreting the results is tracking the information on the atoms while the model is running in order to check if the model is working logically (i.e.

Table 2. End-to-end donation times of the observed data and the two models.

\begin{tabular}{cccc}
\hline $\begin{array}{c}\text { Type of } \\
\text { donation }\end{array}$ & Reality (observed data) & $\begin{array}{c}\text { First model (queue atom } \\
\text { before actual donation) }\end{array}$ & $\begin{array}{c}\text { Second model (multi-service } \\
\text { atom before actual donation) }\end{array}$ \\
\hline Blood & $\begin{array}{c}17 \text { minutes } \\
\text { (estimation of } 6 \text { minutes) }\end{array}$ & 21.2 minutes & 21.3 minutes \\
Plasma & 57.7 minutes & 60.7 minutes & 65.3 minutes \\
Platelets & 95 minutes & 91.6 minutes & 98.5 minutes \\
\hline
\end{tabular}


model verification). The ArrivalList atom represents the number of arriving donors and the Sink atoms mention the number of donors, separated by the type of donation, who leave the flow and have a drink in the donor corner. Furthermore, the Server atoms indicate the utilization rate of the servers, which is the ratio of the busy time and the total time. For example, the doctor is examining donors for $60.87 \%$ on average of his/her time during a simulation run of 12 hours (see Figure 4). While running the simulation model, the utilization rates are increasing during peak times and decreasing during the less congested hours. The Queue and Multi-Service atoms are also changing during the run, showing the number of donors that are waiting or being served in the corresponding step.

Results can also be obtained by connecting monitors (e.g. bar graphs, status pies, etc.) to atoms. As shown in Figure 4, the status pie 'Status Registration' indicates that the person at the registration desk is busy $40 \%$ of the total time. The monitor 'Queue Registration', connected to the entering queue, shows that the arriving donors are waiting on average 52 seconds before being served by the person at the registration desk. After being registered, donors are using on average 1.3 out of five spots to answer the medical questionnaire. Furthermore, donors are waiting on average 1.7 minutes in order to get assigned to a plasma bed, while there is no average waiting time for donation of blood and platelets. The monitoring of the occupation of the beds reveals an underutilization as only $0.3,3.8$ and 1.8 beds are utilized out of 3, 7 and 4 beds respectively for blood, plasma and platelets.

Thirdly, results can be interpreted by retrieving a summary report (see Figure 5), which represents an overview of the donor flow's status and provides feedback on the impact of changing the input parameters. The average content in Figure 5 indicates possibilities for optimizing the resource utilization. For example, on average 1.276 spots (out of 5 spots) are used to complete the medical questionnaire. This result is similar to the monitors in Figure 4. The average content in a queue determines how many donors are waiting. Notably, the queues are not so large. On average, donors are waiting one minute before a bed becomes available for donating plasma, while there are no waiting

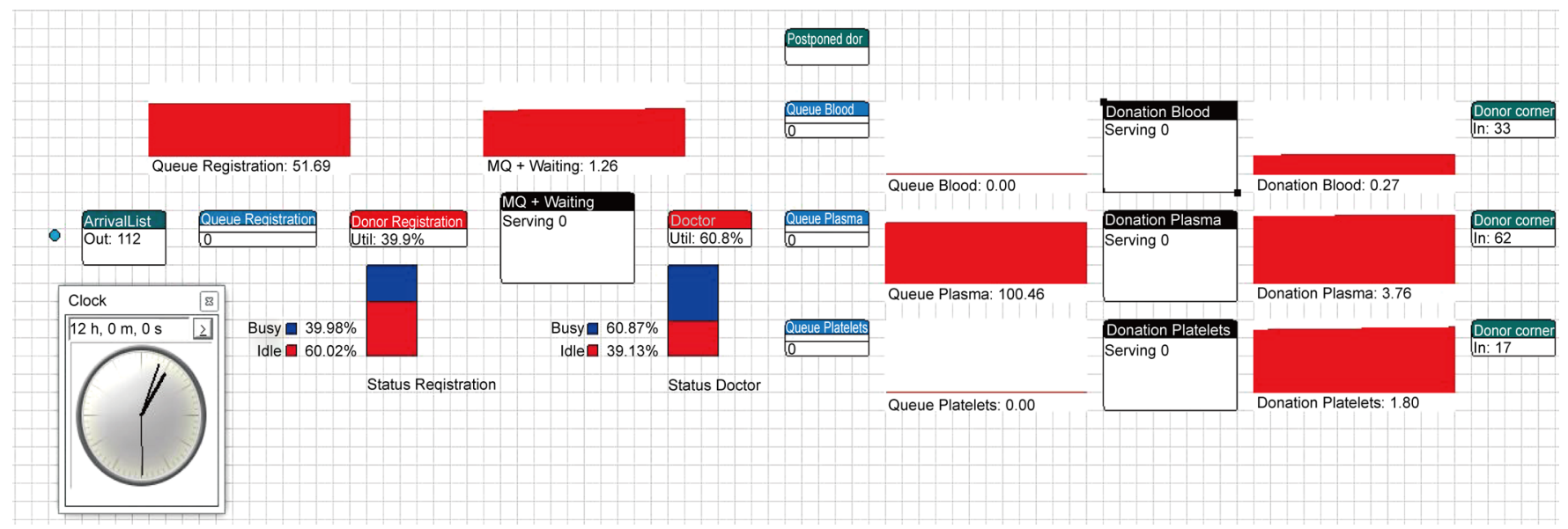

Figure 4. Results after a 12-hour run of the first simulation model. 


\begin{tabular}{|c|c|c|c|c|c|}
\hline \multirow[b]{2}{*}{ name } & \multicolumn{2}{|c|}{ content } & \multicolumn{2}{|c|}{ throughput } & \multirow{2}{*}{$\begin{array}{l}\text { staytime } \\
\text { average }\end{array}$} \\
\hline & current & average & input & output & \\
\hline Donorregistrati & 0 & 0.391 & 112 & 112 & 150.906 \\
\hline Donor corner & 0 & 0.000 & 33 & 0 & 0.000 \\
\hline Doctor & 0 & 0.609 & 112 & 112 & 234.728 \\
\hline Postponed & 0 & 0.000 & 0 & 0 & 0.000 \\
\hline MVL + Waiting & 0 & 1.276 & 112 & 112 & 492.274 \\
\hline Donation Blood & 0 & 0.390 & 33 & 33 & 511.108 \\
\hline Donation Plasma & 0 & 3.473 & 62 & 62 & 2419.840 \\
\hline Donation Platel & 0 & 1.889 & 17 & 17 & 4800.117 \\
\hline Sink25 & 0 & 0.000 & 62 & 0 & 0.000 \\
\hline Sink26 & 0 & 0.000 & 17 & 0 & 0.000 \\
\hline Queue donorregi & 0 & 0.125 & 112 & 112 & 48.343 \\
\hline Product & 0 & 0.000 & 0 & 0 & 0.000 \\
\hline ArrivalList & 0 & 0.000 & 112 & 112 & 0.000 \\
\hline Queue Blood & 0 & 0.000 & 33 & 33 & 0.000 \\
\hline Queue Plasma & 0 & 0.062 & 62 & 62 & 42.858 \\
\hline Queue Platelets & 0 & 0.000 & 17 & 17 & 0.000 \\
\hline
\end{tabular}

Figure 5. Output of the summary report for the first model.

times for donating blood or platelets. In the observed data, however, the waiting times were longer, as measured in the second simulation model.

The three above mentioned methods are particularly useful to display results directly during the simulation run, but these are less appropriate when making decisions based on the results of the model. These techniques are primarily utilized for building and testing the model (i.e. model verification and validation in section 4.4), while the fourth measuring technique, experimentation, is used later on in the process when the model is more reliable. In this case, an experiment will be conducted in which performance measures (e.g. maximum waiting time in each step, maximum utilization of beds, etc.) can be defined. The experiment was set to execute ten separate runs of 12 hours.

Table 3 presents the results from the experiment, which are comparable to the results obtained above. The results will be discussed now, and the next section provides some recommendations.

A donor is spending on average 52 seconds in the entering queue. In peak hours, however, the waiting time can increase to 6 minutes on average for the ten observations. In the donor centers, five spots are arranged for completing the medical questionnaire, while only 1.3 of the spots are used on average. When observing the queues before being assigned to a bed, donors are spending on average 1.3 minutes in the waiting line for donating plasma, while they do not have to wait to donate blood or platelets. During peak hours, donors are waiting maximally 13.3 minutes for plasma donations. In the second simulation model the donors are forced to wait for some time (see Table 2 with probability distributions), even if beds are available. The experiment wizard of the second model reports waiting times of on average 6.3 minutes for platelets and 5.1 minutes for plasma, which is much more than the 1.3 minutes for plasma in the first model. These time differentials between the two models could be explained by the 
Table 3. Output of experiment wizard of the first model.

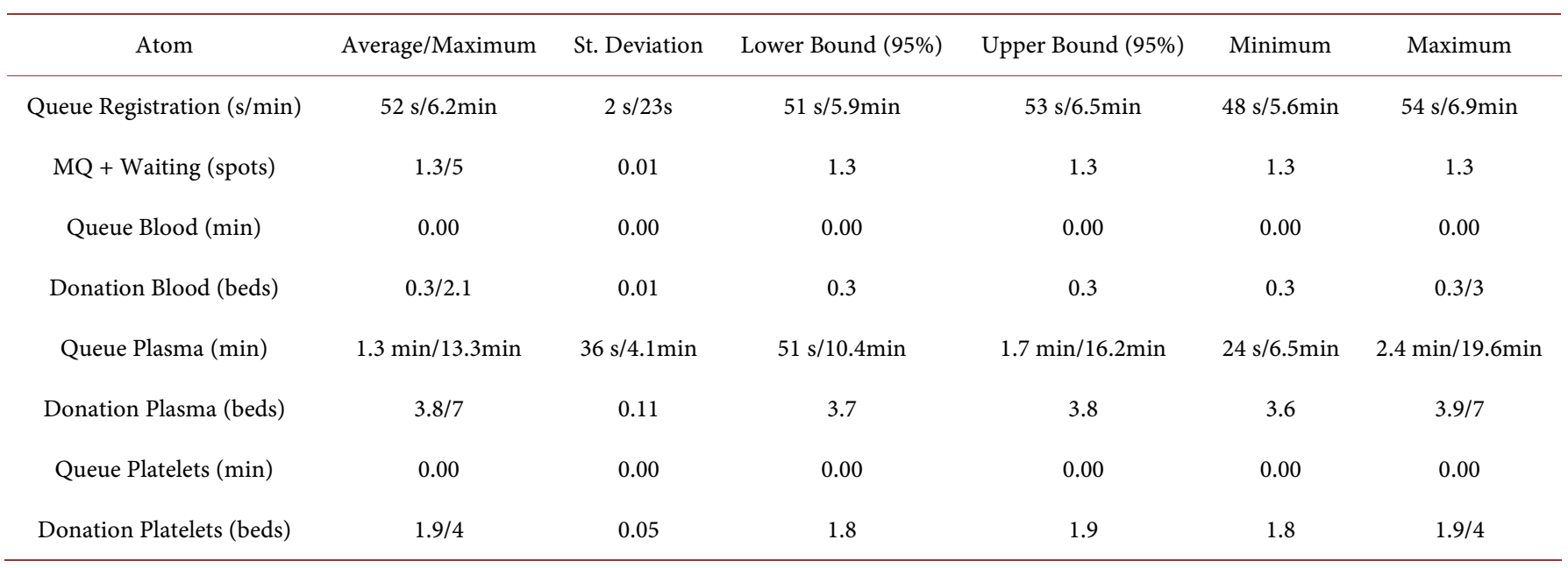

personnel occupation (i.e. human resource team). Finally, the actual donation time differs between the three types of donations, as well as the number of beds. The blood donation atom has a capacity of three beds and an average content of 0.28 beds utilized. Plasma donation has a capacity of seven beds in the donor center, while the average content is only 3.8 beds utilized. For the donation of platelets, four beds are available in the donor center from which 1.9 beds are utilized. This result illustrates that, on average, there is underutilization of the beds. Although, in peak hours, the beds are maximally utilized.

\subsection{Recommendations}

The results of the experiments show that simulation is useful for a better understanding of the current donor flow. Moreover, the visualization of the flow can be a useful tool to convince the medical experts of the improvements that can be made by adapting the capacity of the donor center to the expected number of arriving donors, and hence decreasing the waiting time. By analyzing the results obtained in the two simplified models, recommendations can be made. The purpose of these recommendations is identifying the gaps of information: in order to build a more detailed simulation model for testing different scenarios and optimizing the donor flow, more data will be needed. In this gap analysis, it will become clear which additional data should be collected or extracted from available reports within the Red Cross-Flanders. When more data are available, suggestions for improvements can be made in several realistic scenarios by changing the input parameters.

Donors arriving at the donor center wait in the entering queue. However, some of them have an appointment for their donation and do not have to wait in this queue. When donating plasma or platelets, it is recommended to make an appointment because these donations have a longer duration. To implement two queues (i.e. for donors with/without appointment) in the model, data on the percentage of donors that have an appointment should be collected. This information will also be very useful for the sche- 
duling of personnel as it creates a better knowledge on the inflow of donors to the donor centers. However, as donating blood is a voluntary activity which can be done when it suits the donor best, one should be careful to instigate donors to make an appointment. Another recommendation could be distinguishing between new and experienced donors. A new donor is spending more time at the registration desk than an experienced donor, because he/she receives more information and asks questions about the donor flow. Analyzing the end-to-end donation time of these two types of donors will indicate whether it is advantageous to make separate queues. It is important to collect new data for the time spent at the registration desk in order to build the more detailed model, because the currently observed data are not very reliable as they represent the time that the medical questionnaire is printed, which is often at the end of the registration step.

A third recommendation to the simplified model is splitting the Multi-Service atom "MQ + waiting", which consists of answering the medical questionnaire and the waiting time in front of the medical examination at the doctor's office. In the current model, these two activities are merged together because the available data include the time at which the donor starts filling in the questionnaire and the time at which the donor enters the doctor's office. This creates a bias in the waiting time in our model. The Multi-Service atom for completing the questionnaire should therefore be followed by a Queue atom, and data should be collected on the duration for completing the questionnaire. In this way, the end-to-end donation time could be reduced and a potential bottleneck could be identified.

The number of personnel varies during the day because of lunch breaks, phone calls, administrative tasks, bathroom breaks, etc. As a consequence, the employees are not available to serve the donors for $100 \%$ of their time. The utilization rate of the resources in the first model is not accurate because the available staff has been ignored. In the more detailed model, the time that employees are not working with donors directly (i.e. down time) should be included in the model. In Enterprise Dynamics, additional staff (Human Resource Team) can be added to analyze the impact of changing the capacity of the personnel. In the actual donation step, the nurses and assistants should be assigned to the right beds and donors as this might impact the queue in front of the actual donation. For example, when a donation bed is available, the donor goes to this bed in the model. In reality, however, a nurse is needed to assist the donor to the bed and to prepare the bed for the next donation. Furthermore, it is recommended to collect additional data in the actual donation step. The durations for blood, plasma and platelets donations are ending when the donation set is removed from the donor. Hence, no data are available on the times that the donor leaves the bed (and the bed becomes available for preparing it for the next donation), which is needed to maximize the utilization of the beds.

At the doctor's office, the donor selection includes an eligibility assessment involving a medical questionnaire, an interview and physical examination (pulse, weight and blood pressure). Recently, a hemoglobin screening was implemented for new donors 
and donors who had a low hemoglobin level the last time they donated. The Server atom at the doctor's office in the simplified model contains data in which the hemoglobin screening is included versus not included. In order to obtain a reliable model, the actual percentage of hemoglobin screenings should be compared with the ratio of screenings in the observed data. Another recommendation when building the medical examination atom is to make a connection to the postponed donors Sink atom. These donors do not fulfill the health requirements and are refused to continue the donation (i.e. they leave the flow after step 3). It is important to identify the postponed donors, because they increase the end-to-end donation time of all donors as they also utilize the donor flow until the medical examination. To be able to dispose the right amount of donors, data should be collected to indicate the disposed percentage in the atom.

By collecting the additional data and implementing them in the model, the model will become more reliable and potential bottlenecks may be identified. Different scenarios, such as building two queues (appointments versus no appointments), changing the capacity of the resources, etc. can be tested and compared. In the end, a dynamic planning tool will be developed based on which the Blood Service can adapt the capacity of the donor center to the expected number of donors in order to get the optimized donor flow which decreases the waiting times for donors and increases the productivity of the resources.

\section{Conclusions}

The overall aim of the research presented is to optimize the operations flow at the donor centers of the Red Cross-Flanders in order to improve customer (donor) service and the donor center productivity or utilization. Since this project is still ongoing, this paper only presents a first simplified simulation model, which visualizes the current flow at the donor center. A gap analysis is conducted in which the missing data are identified that are needed to develop a more detailed simulation model. Several recommendations are made which will be tested in different scenarios in order to find the optimized flow at the Red Cross-Flanders.

Decision makers can make better and less risky decisions regarding changes in use of human and material resources on the knowledge created by simulation experiments. There are some limitations in our approach. Models are simplifications and sometimes it is difficult to guarantee that they will be valid. Simulation calls for special expertise and a detailed knowledge of the system being depicted is necessary. In the future, a dashboard will be developed, based on the scenarios tested in the detailed simulation model, which the Red Cross-Flanders can rely on when setting up the capacity of the donor center in terms of both personnel and beds.

\section{References}

[1] Vlaanderen, R.K. What Do We Do? http://www.rodekruis.be/en/what-do-we-do/

[2] Rytilä, J.S. and Spens, K.M. (2006) Using Simulation to Increase Efficiency in Blood Supply Chains. Management Research News, 29, 801-819. 
http://dx.doi.org/10.1108/01409170610717826

[3] Beliën, J. and Forcé, H. (2012) Supply Chain Management of Blood Products: A Literature Review. European Journal of Operational Research, 217, 1-16. http://dx.doi.org/10.1016/j.ejor.2011.05.026

[4] Kendall, K.E. (1984) Planning for Innovation in Health Care: A Breakthrough in Blood Banking. Long Range Planning, 17, 127-131. http://dx.doi.org/10.1016/0024-6301(84)90225-5

[5] Vlaanderen, R.K. Kerncijfers Bloedinstelling. http://www.rodekruis.be/jaarverslag/2014/kerncijfers/\#horizontalTab2

[6] Nicholas, J.M. (2012) An Integrated Lean-Methods Approach to Hospital Facilities Design. Hospital Topics, 90, 47-55. http://dx.doi.org/10.1080/00185868.2012.679911

[7] Womack, J. and Miller, D. (2005) Going Lean in Health Care. Institute for Healthcare Improvement.

[8] Sugimori, Y., Kusunoki, K., Cho, F. and Uchikawa, S. (1977) Toyota Production System and Kanban System: Materialization of Just-in-Time and Respect for Human Systems. International Journal of Production Research, 15, 553-564. http://dx.doi.org/10.1080/00207547708943149

[9] D’Andreamatteo, A., Ianni, L., Lega, F. and Sargiacomo, M. (2015) Lean in Healthcare: A Comprehensive Review. Health Policy, 119, 1197-1209.

http://dx.doi.org/10.1016/j.healthpol.2015.02.002

[10] Hicks, C., McGovern, T., Prior, G. and Smith, I. (2015) Applying Lean Principles to the Design of Healthcare Facilities. International Journal of Production Economics, 170, 677-686. http://dx.doi.org/10.1016/j.ijpe.2015.05.029

[11] Bohmer, R.M.J. and Ferlins, E.M. (2005) Virginia Mason Medical Center. Harvard Business School Publishing, Boston.

[12] Coletta, A.R. (2012) The Lean 3P Advantage: A Practitioner's Guide to the Production Preparation Process. CRC Press, Boca Raton. http://dx.doi.org/10.1201/b11811

[13] Vlaanderen, R.K. Activiteiten Dienst Voor Het Bloed. http://www.dienstvoorhetbloed.be/DVB-Channel/sites-Dienst-voor-Bloed-Nederlands/Ove r-Dienst-voor-het-Bloed/Activiteiten-Dienst-voor-het-Bloed.html

[14] Shannon, R.E. (1998) Introduction to the Art and Science of Simulation. Proceedings of the 1998 Winter Simulation Conference, 7-14. http://dx.doi.org/10.1109/wsc.1998.744892

[15] Chemweno, P., Thijs, V., Pintelon, L. and Van Horenbeek, A. (2014) Discrete Event Simulation Case Study: Diagnostic Path for Stroke Patients in a Stroke Unit. Simulation Modelling Practice and Theory, 48, 45-57. http://dx.doi.org/10.1016/j.simpat.2014.07.006

[16] In Control Simulation Solutions. Enterprise Dynamics. http://www.incontrolsim.com/en/enterprise-dynamics/enterprise-dynamics-overview.html

[17] Roberts, D.S. (2011) Tutorial on the Simulation of Healthcare Systems. North Carolina State University Edward P. Fitts, Department of Industrial and Systems Engineering.

[18] Schlesinger, S., et al. (1979) Terminology for Model Credibility. Simulation, 32, 103-104. http://dx.doi.org/10.1177/003754977903200304

[19] Sargent, R.G. (2007) Verification and Validation of Simulation Models. Proceedings of the 2007 Winter Simulation Conference, 124-137. http://dx.doi.org/10.1109/wsc.2007.4419595 
Submit or recommend next manuscript to SCIRP and we will provide best service for you:

Accepting pre-submission inquiries through Email, Facebook, LinkedIn, Twitter, etc. A wide selection of journals (inclusive of 9 subjects, more than 200 journals)

Providing 24-hour high-quality service

User-friendly online submission system

Fair and swift peer-review system

Efficient typesetting and proofreading procedure

Display of the result of downloads and visits, as well as the number of cited articles

Maximum dissemination of your research work

Submit your manuscript at: http://papersubmission.scirp.org/

Or contact ajibm@scirp.org 